Sharif University of Technology
Scientia Iranica
Transactions E: Industrial Engineering
http://scientiairanica.sharif.edu

\title{
Determining optimal machine part replacement time using a hybrid ANN-GA model
}

\author{
S.H. Gökler* and S. Boran \\ Department of Industrial Engineering, Sakarya University, Sakarya, 54050, Turkey.
}

Received 5 February 2019; received in revised form 10 December 2019; accepted 15 June 2020

\section{KEYWORDS}

Replacement time;

Replacement cost model;

Artificial neural

network;

Genetic algorithm;

Hybrid ANN-GA

model.

\begin{abstract}
Companies must determine the replacement time of machine parts correctly since it affects their production costs and efficiencies. In this respect, the objective is to determine the most appropriate replacement time to minimize cost per unit. This study proposes developing a hybrid Artificial Neural Network (ANN)-Genetic Algorithm (GA) model to predict replacement time without using a cost model. At first, a replacement cost model is developed to calculate replacement times to use in training the neural network. Nevertheless, the cost model needs complex mathematical calculations. GA is used instead of the cost model to determine replacement time and thus, to achieve fast learning for the neural network. The hybrid ANN-GA model was applied to predict replacement time of bladder in tire manufacturing. Furthermore, ANN and GA models, which were developed to increase the prediction accuracy of the hybrid model, were used. The hybrid ANN-GA model presented a better solution according to the performance statistics than the other ANN and GA models. The values indicate that the hybrid model is in good agreement with the cost model. Thus, it is recommended that the hybrid model be used instead of the cost model.

(C) 2022 Sharif University of Technology. All rights reserved.
\end{abstract}

\section{Introduction}

As the costs associated with machine parts correspond to a large proportion of the total cost of production, economical machine part replacement times are very important, especially for expensive parts [1]. Optimal replacement time should be obtained to ensure minimization of the expected average cost per unit time. Replacement cost models function based on the economic comparison between planned (preventive) and unplanned (failure) replacement actions. In planned replacement, machine parts may be changed at only scheduled times and in this case, these parts might not have completed their useful life. In unplanned

*. Corresponding author. Tel.: 90-3443001000 E-mail addresses: shgokler@sakarya.edu.tr (S.H. Gökler); boran@sakarya.edu.tr (S.Boran)

doi: $10.24200 /$ sci. 2020.52828 .2902 replacement, replacement is made in the case of failed machine parts during operations. This case may damage the product which is processed on the machine and therefore, causes additional scrap product costs.

Machine part replacement time is regarded as a random variable that is usually modeled by Weibull distribution in replacement cost models. Weibull distribution is the most widely used probability distribution of reliability studies because it is highly flexible in compliance with random data and has the ability to be adopted for data with different distributions [24]. In a replacement cost model, Weibull distribution parameters, $\alpha$-scale and $\beta$-shape, need to be updated with new replacement data to revise replacement strategies [5]. It is very time-consuming and laborintensive to calculate the new replacement time based on the cost model involving complex mathematical operations every time.

This study proposes a hybrid model composed of Artificial Neural Network (ANN) and Genetic Algo- 
rithm $(\mathrm{GA})$ to predict a new replacement time without the need for a mathematical model. The ANN method is used to predict the machine part replacement time, which minimizes the cost per unit. Since the ANN method evaluates not only present data but also past data, it predicts replacement time more accurately than cost models. A replacement time cost model is developed to provide the necessary data for the training of the ANN. The GA method is used instead of the developed cost model to find replacement times and thus, to accelerate the learning of the neural network. The replacement times obtained with the GA method correspond to the output data needed for the training of the neural network.

Replacement time cost model studies in the literature are mostly concerned with machine tool replacement [6-8] and machine replacement [9,10], rather than machine part replacement. A number of studies have been carried out with different criteria than those known in replacement cost models. In one of them, Wang et al. [11] used profit instead of cost as the criterion of economics in their replacement model. In another study, Sheikh et al. [12] used a number of products processed in the machine instead of the lifetime in the cost function to determine the optimal machine tool replacement interval.

Hybrid ANN-GA algorithms were used in the past for the cost minimization problem by researchers. Hashemi et al. [13] proposed a hybrid model including ANN optimized by GA for estimating power plant project costs. ANN was used to predict the costs, whereas GA was used to set the ANN's parameters such as number of hidden layers, number of nodes per each hidden layer, and the corresponding weights and biases. Seo [14] developed a hybrid GA-ANN model to predict product life cycle costs. GA was employed to improve ANN by eliminating irrelevant factors, determining the number of hidden nodes and processing elements, and optimizing the connection weights between layers. Firouzi and Rahai [15] achieved a hybrid ANN-GA model that optimizes risk-based repair and maintenance actions and yields the minimum life cycle cost for concrete bridge decks.

A limited number of studies exist in the literature on the ANN method, GA method, or hybridized ANN and GA methods for determining replacement times. Al-Chalabi et al. [16] presented a model-based ANN method to determine the Economic Replacement Time (ERT) of production machines. Aldhubaib and Salama [17] illustrated an approach to link maintenance and replacement decisions. They adopted GA to optimally schedule maintenance activities. Liu et al. [18] conducted a study using ANN, GA, and Weibull distribution together. They structured a model to determine long-run fuzzy expected replacement cost per unit time and the optimal preventive replacement interval. The ANN method was used for parameter estimation, reliability prediction, and evaluation of the expected maintenance cost. The GA method was employed to find the values for the membership function at any cut level. The effectiveness of the proposed method was illustrated using a two-parameter Weibull distribution.

In the literature, in cost-based hybrid ANN-GA models, GA was mostly used for tuning the parameter values of ANN. In this study, unlike others, GA is used to obtain replacement time, which is the neural network model's outputs based on the cost model.

Application of the developed hybrid model in a real setting was illustrated on a bladder used in a curing press in tire manufacturing. ANN and GA models were individually created to increase the replacement time prediction performance of the developed hybrid model. The application results of the developed hybrid ANN-GA model, GA, and ANN models were separately compared to the results obtained from the proposed replacement cost model. According to the performance statistics such as coefficient of determination $\left(R^{2}\right)$, Mean Absolute Percentage Error (MAPE), and Root Mean Square Error (RMSE), the hybrid ANN-GA model obtained results more similar to the results of the proposed replacement cost model than those of the ANN and GA models. Hence, the hybrid ANNGA model is recommended for predicting machine part replacement time instead of the cost model because it is more convenient and practical, as well.

The contribution of this study may be summarized as follows:

- A machine part replacement cost model was developed;

- A hybrid ANN-GA cost model was structured to predict replacement time;

- It was presented that the hybrid model tended to make a more accurate prediction than the individual prediction models.

\section{Methods}

\subsection{ANN method}

ANN is a machine learning method that can learn a mapping between an input and an output space and synthesize an associative memory that retrieves the appropriate output when new inputs are presented [19]. Neural network is structured with three (input, hidden, and output) layers and the interconnections between the neurons in the layers. Input layer receives features of input data and distributes them to the hidden layers without any processing. While the inputs are transmitted to the hidden layer, the net input of that cell is calculated by transfer function. The hidden layer shows the interactions between input and output layers. 
The net sum obtained from the transfer function is transmitted to the activation function to generate the output of the cell. The output value of the activation function is the output value of the neuron. ANN can have more than one layer and more than one neuron in this layer. The output layer indicating the output nodes of variables shows the output generating according to the input data by processing the information coming from the hidden layer. Then, neural network is trained to minimize the error between the present output and predicted output by the ANN model by adjusting the weights in a neural network. It is utilized based on performance criteria in order to validate the performance of the developed ANN model.

\subsection{GA method}

GA is a heuristic search technique that tries to obtain global solutions using random search techniques as opposed to local solutions, introduced by Holland [20]. It includes various biological terms such as the population or the selection, crossover, and mutation operators [21]. The individual (candidate) solutions and the individual traits of the chromosome are called chromosomes and genes, respectively. GA algorithm begins by determining the parent chromosomes formed by random partitioning of the population into the pairs of chromosomes. Then, crossover operator is employed to produce more improved children chromosomes from the initial parent chromosomes. The process is evaluated by the fitness function, which reflects the goal of the optimization problem. Subsequently, the mutation procedure is applied to the children chromosomes to avoid not falling into a local optimum. In each iteration, the parent and children chromosomes are combined and the best chromosomes from them are selected to update the current population.

\subsection{Integration of $A N N$ with $G A$}

ANN has several disadvantages such as long training time, unwanted convergence to local optimal solution instead of global, and having a large number of parameters. Therefore, it is requested to integrate ANN with another algorithm that can eliminate one of specific problems. An algorithm that has frequently been hybridized with ANN is GA. Recently, the trend to hybridize GA and ANN has been getting common among researchers [13-15]. The advantage provided by forming an ANN-GA hybrid model is to make a more accurate and fast prediction. ANN uses past data to predict future trend, while GA finds the better sets of input variables and input subsets for improving ANN training.

\subsection{Model performance criteria}

The prediction ability of the models can be usually evaluated based on the statistical performance criteria namely $R^{2}$, MAPE, and RMSE. $R^{2}$, RMSE, and
MAPE can be calculated by Eqs. (1), (2), and (3), respectively:

$$
\begin{aligned}
& R^{2}=\left[\frac{\sum_{i=1}^{N}\left(\left(T_{i}-\bar{T}\right)\left(P_{i}-\bar{P}\right)\right)}{\sqrt{\sum_{i=1}^{N}\left(T_{i}-\bar{T}\right)^{2} \sum_{i=1}^{N}\left(P_{i}-\bar{P}\right)^{2}}}\right]^{2}, \\
& R M S E=\sqrt{\frac{1}{N} \sum_{i=1}^{N}\left(T_{i}-P_{i}\right)^{2}} \\
& \operatorname{MAPE}(\%)=\frac{1}{N}\left[\sum_{i=1}^{N} \frac{\left|T_{i}-P_{i}\right|}{T_{i}}\right] \times 100
\end{aligned}
$$

where $T_{i}$ is the $i$ th period part life, $\bar{T}$ is mean part life, $P_{i}$ is the $i$ th period predicted part life, and $\bar{P}$ is the $i$ th period predicted mean part life.

\section{Proposed hybrid ANN-GA model}

The objective is to develop a hybrid ANN-GA model that makes the replacement cost per unit the minimum so as to predict the machine part replacement time. This study consists of three phases. In the first phase, a machine part replacement cost model was developed to determine replacement time minimizing cost per unit. However, since the cost model is stochastic, calculating replacement time is cumbersome and time-consuming. Therefore, in the second phase, GA model was developed instead of cost model to obtain replacement time. In the third phase, the ANN model was created to determine the machine part replacement time without the need for the cost model. The replacement time obtained with GA was used in neural network training.

At the end of each replacement period, it is needed to revise $\alpha$ and $\beta$ parameters of Weibull distribution in the light of the data observed. It is difficult and timeconsuming to determine replacement time with the replacement cost model containing a complex function. In addition, the limited number of existing replacement time data is used to determine time. Since ANN learns from the past data, the number of data that used for prediction is more than the proposed replacement cost model. The hybrid model determines more precise approximate replacement time. The developed hybrid ANN-GA model is shown in Figure 1.

\subsection{Replacement cost model}

The proposed machine part replacement cost model was structured based on the developed replacement cost models by Barlow and Hunter [22], Handlarski [23], and Ahmad and Kamaruddin [24]. Although the cost model is similar to other models in terms of cost components, there are some assumptions that make the former model different. These assumptions are as follows: 


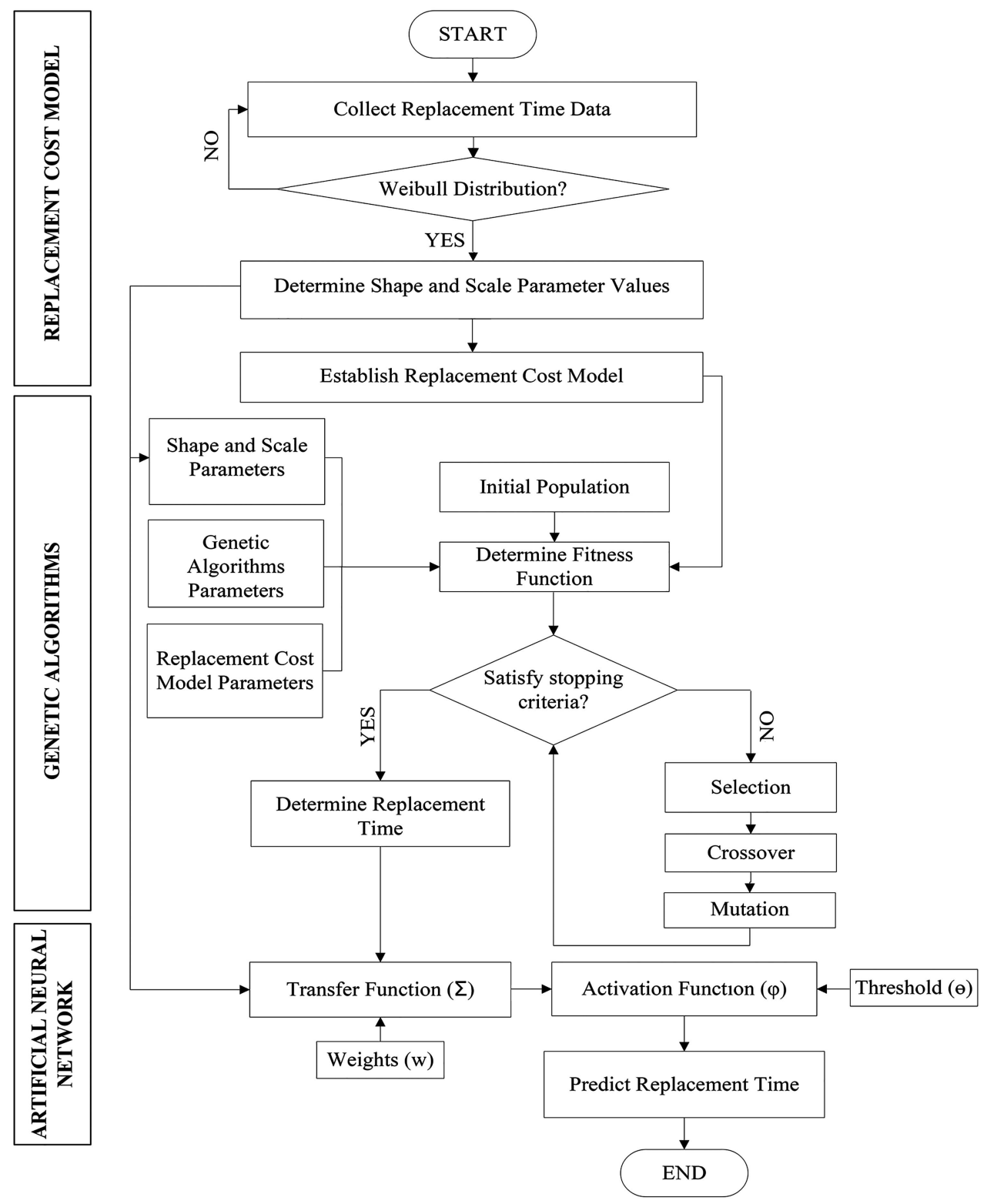

Figure 1. Flowchart of the proposed hybrid ANN-GA model.

- Only one of the machine parts is considered;

- If the machine part fails while the product is processed, it causes product to be subjected to damage and scrap;

- When the machine is stopped to replace a new machine part, the parallel other machine that works together also stops.

The model consists of the planned or unplanned replacement costs. The decision variable of the model is the machine part lifetime. Prior to the introduction of a detailed description of the cost model, some notations are given in Table 1.

\subsubsection{Planned replacement cost}

The planned replacement cost $\left(C_{p}\right)$ is incurred when the machine part is changed upon the completion of its life at the prescribed time. This cost consists of planned loss production cost $\left(C_{l p}\right)$, planned manning cost $\left(C_{m p}\right)$, and replaced part cost $\left(C_{r}\right)$. The loss production cost and manning cost are calculated using Eqs. (4) and (5), respectively:

$$
\begin{aligned}
& C_{l p}=\frac{M C_{c} t_{r}}{t}, \\
& C_{m p}=\frac{C_{e}\left(t_{r}+t_{p}\right)}{60} .
\end{aligned}
$$

The sum of these costs gives the planned replacement cost (Eq. (6)):

$$
C_{p}=\frac{M C_{c} t_{r}}{t}+\frac{C_{e}\left(t_{r}+t_{p}\right)}{60}+C_{r}
$$


Table 1. Notations of the cost model.

\begin{tabular}{|c|c|c|}
\hline Notation & Cost elements & Unit \\
\hline$P$ & Failure detection performance & Scrap product/part failure \\
\hline$C_{s}$ & Product scrap unit value & $€$ \\
\hline$C_{c}$ & Conversion cost & $€$ \\
\hline$T$ & Cycle time & Min \\
\hline$M$ & Machine cavities empty machine & 1,2 \\
\hline$C_{e}$ & Compensation & Cost/hr. \\
\hline$t_{d}$ & Machine down time (waiting time) & Min \\
\hline$t_{r}$ & Replacing time & Min \\
\hline$t_{p}$ & Preparing time planned & Min \\
\hline$t_{u p}$ & Preparing time unplanned & Min \\
\hline$C_{r}$ & Replaced parts cost & $€$ \\
\hline$C_{l p}$ & Loss of production cost (planned replacement) & $€$ \\
\hline$C_{m p}$ & Manning cost (planned replacement) & $€$ \\
\hline$C_{p}$ & Planned replacement cost & $€$ \\
\hline$C_{\text {sup }}$ & Scrap product cost (unplanned replacement) & $€$ \\
\hline$C_{\text {lup }}$ & Loss of production cost (unplanned replacement) & $€$ \\
\hline$C_{m u p}$ & Manning cost (unplanned replacement) & $€$ \\
\hline$C_{u p}$ & Unplanned replacement cost & $€$ \\
\hline$C_{U}$ & Average cost per unit & $€$ \\
\hline$F(x)$ & Cumulative probability function of Weibull distribution & \\
\hline$E(x)$ & Arithmetic mean of Weibull distribution & \\
\hline$\alpha$ & Scale parameter of Weibull distribution & \\
\hline$\beta$ & Shape parameter of Weibull distribution & \\
\hline
\end{tabular}

\subsubsection{Unplanned replacement cost}

Unplanned replacement cost $\left(C_{u p}\right)$ occurs when the machine part suddenly failed during processing on product. Thus, its useful life is shorter than predicted. Unplanned part replacement is usually more timeconsuming than planned replacement. In addition, as the two machines are working in parallel, the breakdown of one machine causes the parallel machine to be out of order. $C_{u p}$ is obtained by adding scrap product cost $\left(C_{\text {sup }}\right)$, unplanned lost production cost $\left(C_{\text {lup }}\right)$, and unplanned manning cost $\left(C_{\text {mup }}\right)$, which are calculated using Eqs. (7), (8), and (9), respectively:

$$
\begin{aligned}
& C_{\text {sup }}=P C_{s}, \\
& C_{\text {lup }}=\frac{M C_{c}\left(t_{d}+t_{r}\right)}{t}, \\
& C_{\text {mup }}=\frac{C_{e}\left(t_{r}+t_{\text {up }}\right)}{60} .
\end{aligned}
$$

$C_{u p}$ is determined using Eq. (10):

$$
C_{u p}=P C_{s}+\frac{M C_{c}\left(t_{d}+t_{r}\right)}{t}+\frac{C_{e}\left(t_{r}+t_{u p}\right)}{60}+C_{r} .
$$

According to all these values, the average cost per unit $\left(C_{U}\right)$ is given as in Eq. (11):

$$
C_{U}=\frac{C_{p}+\left(C_{u p}-C_{p}\right) F(x)}{x(1-F(x))+E(x)} .
$$

$F(x)$ and $E(x)$ values of Weibull distribution in Eq. (11) are calculated using Eqs. (12) and (13), respectively. $x$ is the machine part replacement time or lifetime.

$$
\begin{aligned}
& F(x)=1-e^{-\left(\frac{x}{\alpha}\right)^{\beta}} \quad 0 \leq x \leq \infty, \quad \alpha, \beta \geq 0, \\
& E(x)=\alpha \int_{0}^{\infty} e^{-x} x^{n-1} d x(1+1 / \beta)
\end{aligned}
$$

Maximum likelihood estimation method can be used to calculate $\alpha$-scale and $\beta$-shape parameters of the Weibull distribution (Eqs. (14) and (15)):

$$
\begin{aligned}
& \alpha=\frac{\sum_{i=1}^{n} x_{i}^{\beta}}{n}, \\
& f(\beta)=\left[\frac{\sum_{i=1}^{n} x_{i}^{\beta} \ln x_{i}}{\sum_{i=1}^{n} x_{i}^{\beta}}\right]-1 / \beta-1 / n \sum_{i=1}^{n} \ln x_{i} .
\end{aligned}
$$




\section{Implementation of hybrid ANN-GA model}

The proposed hybrid ANN-GA model was applied to predict bladder replacement time, which is part of a curing press in a tire manufacturing company located in Turkey. The bladder is inflated with pressure in the tire during the change of the green tire in the mold, thereby ensuring that the tire takes shape of mold. Shaping method of tire curing depends on bladder with high elasticity and low rigidity to determine tire cavity profile [25]. Therefore, the bladder is considered as an important part of the curing process. The life and price of purchased bladder vary according to the manufacturer and model. If the predicted bladder life is smaller or larger than the actual value, it yields extra cost. Replacement time of a bladder is the number of tires that bladder has been used in during the curing operation. In other words, bladder lifetime is the number of use of bladder or the number of tires per bladder.

The hybrid ANN-GA model was applied to predict the replacement time of bladder with minimum deviation, which will minimize average cost per tire.

\subsection{Data collection and analysis}

The bladder coded AXYZ was chosen because it is the most used type of bladder in the tire manufacturing company. A damaged bladder is a machine part that will not allow longer use. When the hole, folding, and breakage occur on bladder, its life reaches the end. Bladder's lifetime, which represents the number of tires used during the curing process, is obtained from the past recorded data. Number of tires varies from 1 to 1000 . 1 indicates that the bladder fails in the first tire after being placed on the curing press, whereas 1000 indicates that bladder fails after being used in 1000 tires. Bladder lifetime data were obtained by randomly choosing 30 of the recorded data sets. The data collection process was repeated 120 times. Thus, $3600(120 \times 30)$ replacement time data sets were collected. Anderson-Darling test was applied to determine whether the Weibull distribution of collected data is fitting or not. The values of parameters, $\alpha$ shape and $\beta$-scale, of the Weibull distribution of the data for each period were calculated using Eqs. (14) and (15), respectively. The mean and standard deviation values were measured for each period. The values of the first 3 and last 3 of the 120 periods are given in Table 2 . Minitab 17 was employed to determine whether data fit the Weibull distribution and to obtain values for the parameters of the distribution.

The bladder's average changing time is $13 \mathrm{~min}$. From the observations, the planned preparing time is determined as 5 min on average, while the unplanned preparing time is obtained as $15 \mathrm{~min}$ on average. The average curing time of a tire is $20 \mathrm{~min}$. Due to the fact that the two curing presses operate in parallel with each other, the failure of any bladder causes the other parallel press to stop at the same time. Curing press downtime is $45 \mathrm{~min}$, on average. If the one bladder is damaged, 3 tires become scrappy, on average. The cost of a scrap is an average of $€ 74$ per tire. The unit cost of the AXYZ coded bladder is $€ 94$. The average replacing

Table 2. Replacement times obtained by the cost model.

\begin{tabular}{lccccccc}
\hline \multirow{2}{*}{$\begin{array}{c}\text { Sample } \\
\text { number }\end{array}$} & \multicolumn{7}{c}{ Period } \\
\cline { 2 - 8 } 1 & $\mathbf{1}$ & $\mathbf{2}$ & $\mathbf{3}$ & $\ldots$ & $\mathbf{1 1 9}$ & $\mathbf{1 1 8}$ & $\mathbf{1 2 0}$ \\
2 & 101 & 304 & 447 & $\ldots$ & 410 & 321 & 669 \\
3 & 369 & 112 & 325 & $\ldots$ & 315 & 293 & 442 \\
4 & 525 & 674 & 387 & $\ldots$ & 591 & 468 & 301 \\
5 & 489 & 249 & 267 & $\ldots$ & 577 & 415 & 80 \\
$\ldots$ & 896 & 301 & 505 & $\ldots$ & 385 & 329 & 612 \\
26 & $\ldots$ & $\ldots$ & $\ldots$ & $\ldots$ & $\ldots$ & $\ldots$ & $\ldots$ \\
27 & 544 & 495 & 491 & $\ldots$ & 448 & 484 & 382 \\
28 & 488 & 594 & 125 & $\ldots$ & 443 & 381 & 630 \\
29 & 985 & 798 & 293 & $\ldots$ & 617 & 550 & 451 \\
30 & 222 & 459 & 544 & $\ldots$ & 407 & 662 & 548 \\
Maximum & 720 & 229 & 287 & $\ldots$ & 631 & 236 & 378 \\
Minimum & 985 & 798 & 621 & $\ldots$ & 765 & 791 & 707 \\
Mean & 101 & 112 & 125 & $\ldots$ & 248 & 236 & 80 \\
Standard deviation & 234 & 175 & 123 & $\ldots$ & 118 & 130 & 151 \\
\hline
\end{tabular}


cost of a bladder is $€ 35$ and the compensation cost is $€ 25$ per hour.

\subsection{Determination of replacement time using $G A$}

GA, based on the developed cost model, was provided to determine replacement time for each period to minimize unit cost. These data sets were used for training the ANN model. The equation of average replacement cost per tire, given in Eq. (11), was accepted as the objective function of GA. The GA model is shown in Figure 2.

The parameter values in the GA are given in Table 3. These values were determined through other studies in the literature [26-28] and based on experts' opinions.

\subsection{Prediction of replacement time using $A N N$}

The replacement time obtained from GA and the parameters' values of Weibull distribution were used to train ANN. In the testing phase of the ANN model, the
Table 3. Parameter settings for GA.

\begin{tabular}{lc}
\hline \multicolumn{1}{c}{ Setting type } & Value \\
\hline Encoding scheme & Double vector \\
Population size & 500 \\
Evolution generation & 50 \\
Selection & Roulette wheel \\
Crossover & One point \\
Mutation & Uniform \\
Crossover probability $(\mathrm{Pc})$ & 0.6 \\
Mutation probability $(\mathrm{Pm})$ & 0.01 \\
Generations & 100 \\
\hline
\end{tabular}

parameters of the Weibull distribution are the inputs and the replacement time obtained from GA is the output. The ANN model is as follows (Figure 3 ).

ANN model with feed forward backpropagation consists of 2 inputs and 1 output and it was structured to predict the replacement time. Multi-Layer Perceptron (MLP) model was chosen which was the

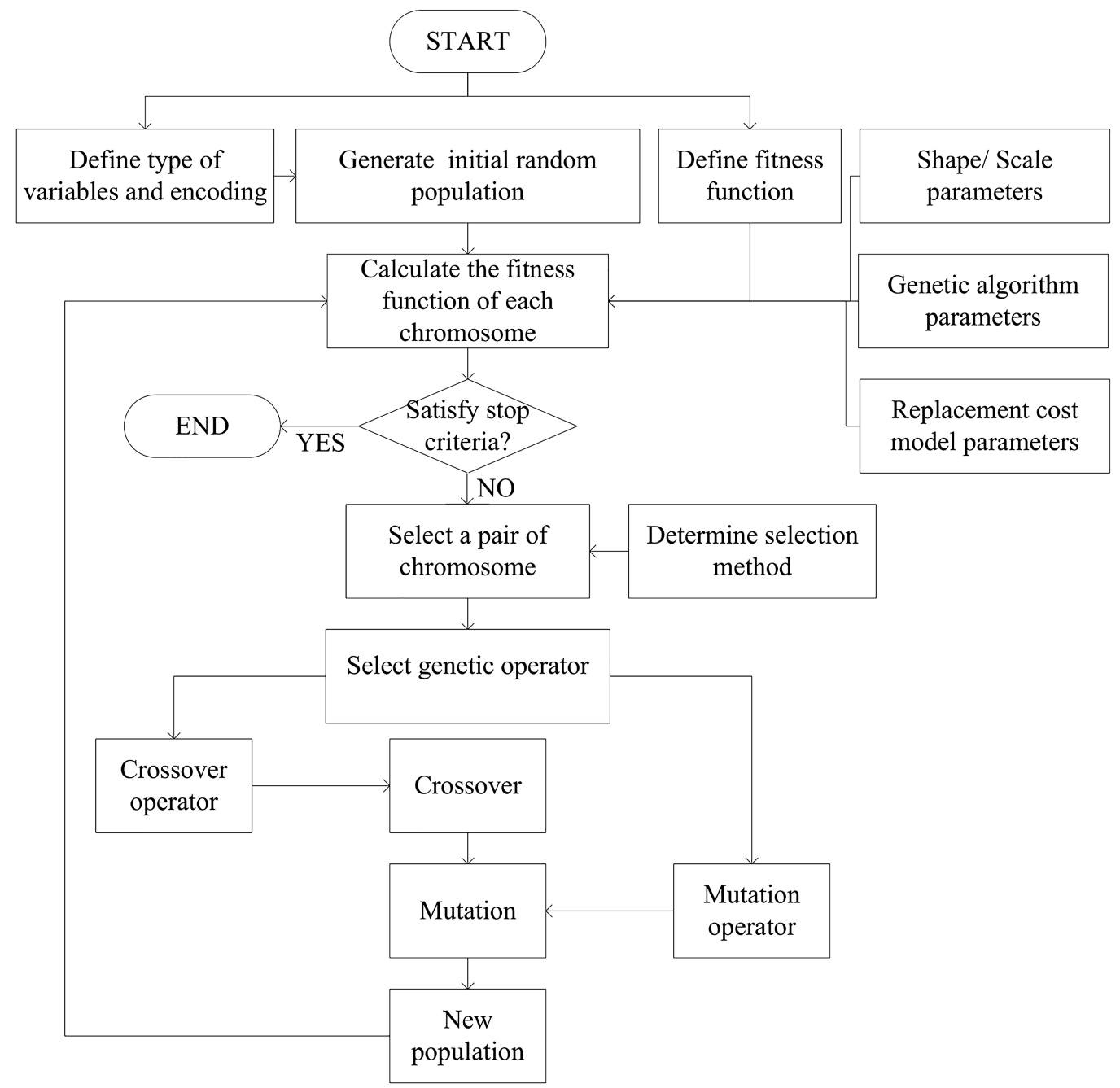

Figure 2. Flowchart of GA model. 


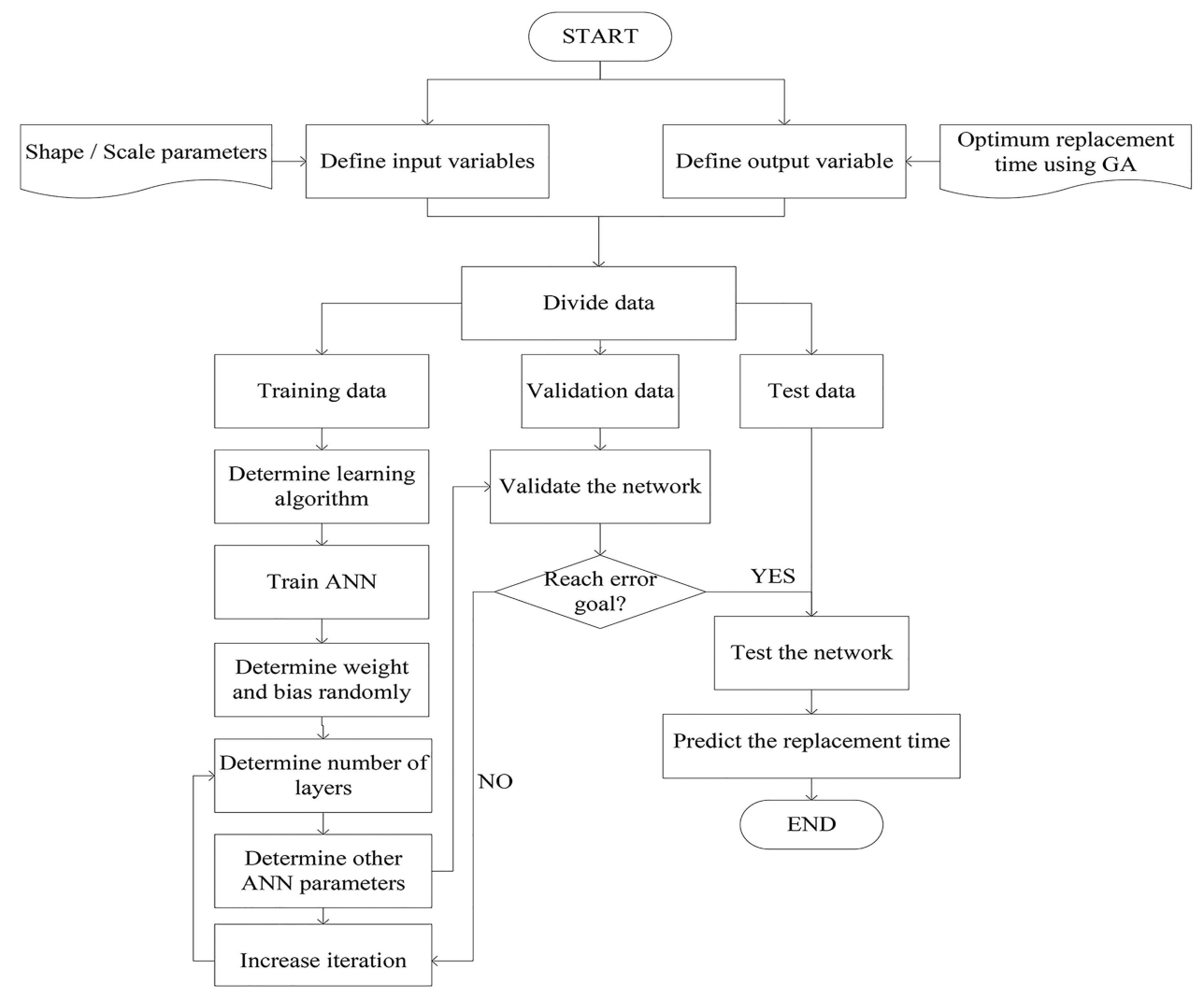

Figure 3. Flowchart of ANN model.

best model for prediction problems [29]. 80\%, 10\%, and $10 \%$ of the data sets were used for training, testing, and validation, respectively.

After determining the initial weight values and threshold values of the inputs, the data in the training set were shown in the network. These datasets were the replacement time belonging to 96 periods $(120 \times 0.80)$. The selected seven training algorithms (Table 4) were tested to determine the best one among them.

The test performance results of each algorithm according to $R^{2}$ are given in Table 5. Traingdx algorithm seems to provide the best performance according to these results.

The number of hidden layers affects ANN performance. Therefore, the neural network was tested in the case of different numbers of layers to determine the most suitable layer number. The results are given in Table 6. From the table, it appears that a single layer provides the best performance for validation and testing with respect to $R^{2}$. This case can be considered as a good result because having more than one hidden layer slows learning, as already explained in the literature [30].

Values for other parameters of the ANN model were determined by the studies existing in the literature [31-33] and based on experts' opinions (Table 7).

Figure 4 shows the ANN model's structure for predicting replacement time. MATLAB12 software was utilized for ANN and GA calculations.

The performance of the network was measured in

Table 4. The ANN training algorithms utilized in this study.

\begin{tabular}{ccl}
\hline Abbreviation & Algorithm & \multicolumn{1}{c}{ Description } \\
\hline CGF & Traincgf & Fletcher-Powell conjugate gradient \\
CGP & Traincgp & Polak-Ribiére conjugate gradient \\
GD & Traingd & Gradient descent \\
GDA & Traingda & Gradient descent with adaptive learning rate \\
GDX & Traingdx & Gradient descent with variable learning rate \\
LM & Trainlm & Levenberg-Marquardt \\
RP & Trainrp & Resilient backpropagation \\
\hline
\end{tabular}




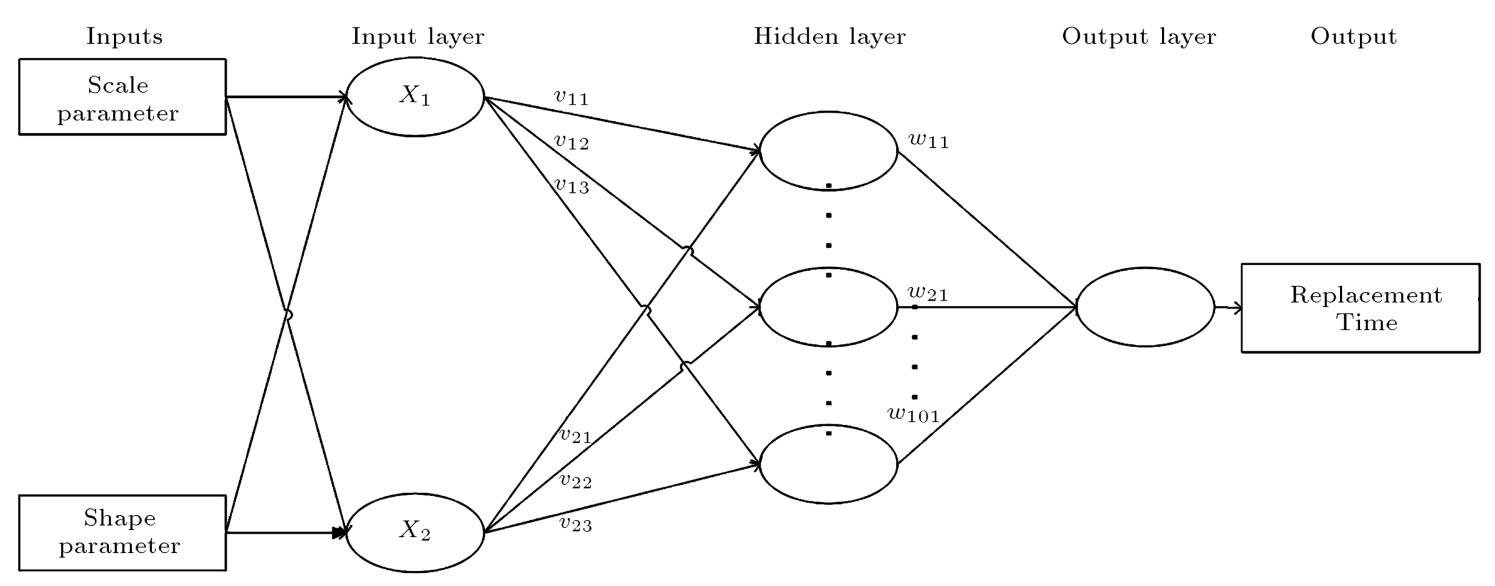

Figure 4. ANN model structure.

Table 5. Performance results for the training algorithms.

\begin{tabular}{lcccc}
\hline \multirow{2}{*}{ Training algorithm } & \multicolumn{3}{c}{$\boldsymbol{R}^{\mathbf{2}}$} \\
\cline { 2 - 5 } & Training & Validation & Test & All \\
\hline CGF (Fletcher-Powell Conjugate Gradient) & 0.803 & 0.933 & 0.774 & 0.817 \\
CGP (Polak-Ribiére Conjugate Gradient) & 0.845 & 0.733 & 0.719 & 0.823 \\
GD (Gradient Descent) & 0.837 & 0.750 & 0.806 & 0.817 \\
GDA (Gradient Descent with Adaptive learning rate) & 0.857 & 0.935 & 0.789 & 0.872 \\
GDX (Gradient Descent with variable learning rate) & 0.939 & 0.953 & 0.943 & 0.943 \\
LM (Levenberg-Marquardt) & 0.935 & 0.924 & 0.884 & 0.924 \\
RP (Resilient Backpropagation) & 0.920 & 0.931 & 0.904 & 0.910 \\
\hline
\end{tabular}

Table 6. Results of ANN performance for different numbers of layers.

\begin{tabular}{lccccc}
\hline Number of layer & $\mathbf{1}$ & $\mathbf{2}$ & $\mathbf{3}$ & $\mathbf{4}$ & $\mathbf{5}$ \\
\hline Training $\boldsymbol{R}^{\mathbf{2}}$ & 0.939 & 0.307 & 0.935 & 0.691 & 0.762 \\
Validation $\boldsymbol{R}^{\mathbf{2}}$ & 0.953 & 0.163 & 0.955 & 0.671 & 0.904 \\
Test $\boldsymbol{R}^{\mathbf{2}}$ & 0.943 & 0.283 & 0.863 & 0.773 & 0.542 \\
All $\boldsymbol{R}^{\mathbf{2}}$ & 0.943 & 0.268 & 0.927 & 0.677 & 0.738 \\
\hline
\end{tabular}

Note: $R^{2}$ : Coefficient of determination

Table 7. Parameters values for the neural network model.

\begin{tabular}{lc}
\hline \multicolumn{1}{c}{ Setting type } & Value \\
\hline Network type & Feed-forward backpropagation \\
Training function & Levenberg-Marquardt \\
Learning rate & 0.4 \\
Momentum rate & 0.7 \\
Total function & Weighted sum \\
Transfer function & Tansig \\
Number of neuron & 10 \\
\hline
\end{tabular}

using the test data set that has not been used before. The network's prediction accuracy exhibits the performance of the neural network model. Twelve (10\%) and Twelve (10\%) out of the 120 periods were used for testing and validation, respectively. Regression graphs related to training, validation, and testing show the similarity between the network output values and the target values (Figure 5). The results can be interpreted as perfect because the values are located on the line with a 45-degree angle. The calculated $R^{2}$ value for all the data sets was about 0.943 . The results indicate that the hybrid model is sufficient to predict machine part replacement time.

The replacement times obtained using ANN model for the first 3 and the last 3 out of 120 periods are shown in Table 8 .

\section{Results and discussion}

ANN and GA models were individually developed to increase their prediction capabilities, thus keeping up with the capability of the hybrid model. The replacement time values that are obtained using proposed cost model were separately compared with that determined by each model for 120 periods. The inputs ( $\alpha$ and $\beta$ parameter' values) and output (replacement time values) of models for the first 3 and last 3 out of 120 periods are given in Table 8 .

The values for the statistical performance criteria of the models are given in Table 9 . The predictive 

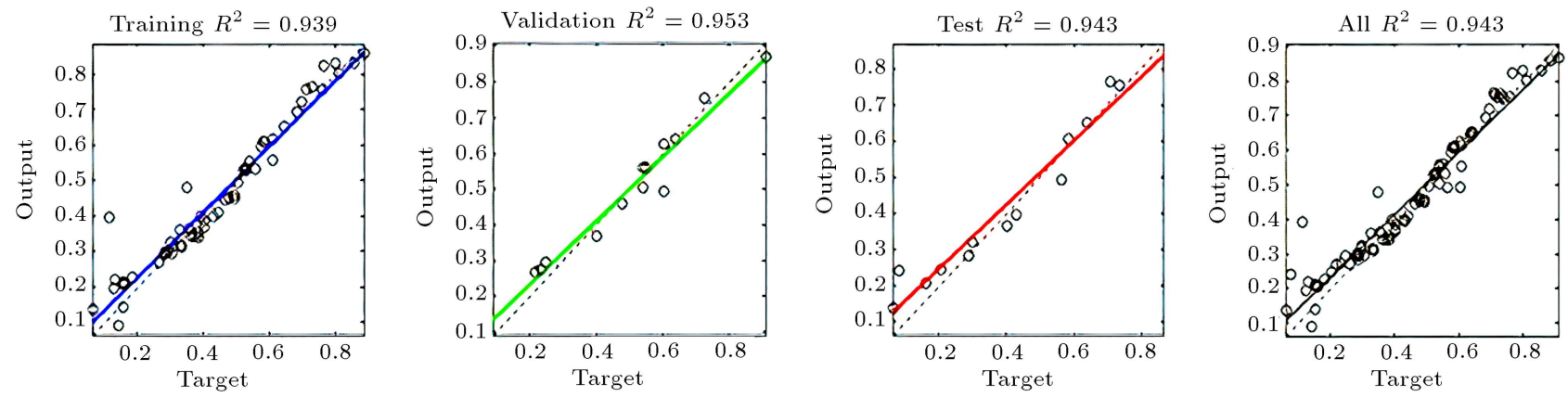

Figure 5. The best performance of ANN.

Table 8. Inputs and outputs of models.

\begin{tabular}{llccccccc}
\hline & $\mathbf{1}$ & $\mathbf{2}$ & $\mathbf{3}$ & $\cdots$ & $\mathbf{1 1 9}$ & $\mathbf{1 1 8}$ & $\mathbf{1 2 0}$ \\
\hline \multirow{2}{*}{ Input } & $\alpha$-scale & 516,33 & 404,94 & 447,94 & $\ldots$ & 609,15 & 517,34 & 486,24 \\
& $\beta$-shape & 2,0521 & 2,2522 & 4,0334 & $\ldots$ & 4,5544 & 2,7818 & 3,1617 \\
& & & & & & & & \\
\multirow{5}{*}{ Output } & Cost model & 325 & 245 & 269 & $\ldots$ & 374 & 301 & 283 \\
& Hybrid ANN-GA & 323 & 220 & 276 & $\ldots$ & 376 & 302 & 288 \\
& GA & 326 & 175 & 260 & $\ldots$ & 378 & 305 & 286 \\
& ANN & 248 & 200 & 234 & $\ldots$ & 286 & 260 & 245 \\
\hline
\end{tabular}

Table 9. Performance comparison of the models.

\begin{tabular}{lccc}
\hline \multicolumn{1}{c}{ Model } & $\boldsymbol{R}^{\mathbf{2}}$ & RMSE & MAPE \\
\hline Hybrid ANN-GA & 0.943 & 9.1240 & 2.5280 \\
GA & 0.908 & 14.760 & 2.9450 \\
ANN & 0.799 & 45.654 & 16.389 \\
\hline
\end{tabular}

Note: $R^{2}$ : Coefficient of determination; MAPE: Mean Absolute Percentage Error; RMSE: Root Mean Square Error.

capabilities of the proposed hybrid ANN-GA, ANN and GA models were compared with that of the replacement cost model. Results indicated that the hybrid ANN-GA model outperformed the other ANN and GA models. Higher $R^{2}$ as well as lower RMSE and MAPE values were obtained by the hybrid model, compared to the other models. The hybrid model proposed in this study is in good agreement with the proposed cost model. The correlation value, $R^{2}$, shows that the model has high explanatory power [34]. Hence, the hybrid ANN-GA prediction data are approximate to the proposed replacement cost model data. The verification analysis confirmed the hybrid model to be highly accurate, reliable, and practical for predicting replacement time. These results are similar to those of the studies in the literature $[35,36]$.

\section{Conclusion}

This study aimed to determine the machine part replacement time that minimized the cost per unit product. The study included three main issues:
- A machine part replacement cost model was developed;

- A hybrid Artificial Neural Network (ANN)-Genetic Algorithm (GA) model was developed to make a prediction of the replacement time without the stochastic replacement cost model requiring complex mathematic operations. The hybrid model would predict a replacement time for the new data set;

- ANN and GA models were separately developed to increase the predictive capability of the hybrid ANN-GA model. The prediction result of each model was compared to that of the proposed replacement cost model based on prediction performance statistics. It was found that the hybrid model's predictive capability was better than the single ANN and GA models.

The developed hybrid ANN-GA model was more convenient and practical than the replacement cost model. Therefore, the hybrid model may be successfully used for determining replacement times. In a future study, the aim should be the use of different machine learning algorithms such as support vector machine, regression trees, and random forest.

\section{References}

1. Jeang, A. "Reliable tool replacement policy for quality and cost", Eur. J. Oper. Res., 108(2), pp. 334-344 (1998). 
2. Nakagawa, T. and Yasui, K. "Calculation of agereplacement with Weibull failuretimes", IEEE Trans Reliab., 30(2), pp. 163-164 (1981).

3. Jonge, B., Klingenberg, W., Teunter, R., et al. "Optimum maintenance strategy under uncertainty in the lifetime distribution", Reliab. Eng. Syst. Safe., 133, pp. 59-67 (2015).

4. Lewis, E.E., Introduction to Reliability Engineering, John Wiley \& Sons Press, Singapore (1987).

5. Mazzuchi, T.A. and Soyer, R. "A Bayesian perspective on some replacement strategies", Reliab Eng Syst Safe., 51, pp. 295-303 (1996).

6. Eryilmaz, S. "Computing optimal replacement time and mean residual life in reliability shock models", CAIE., 103, pp. 40-45 (2017).

7. Xu, W. and Cao, L. "Optimal tool replacement with product quality deterioration and random tool failure", Int. J. Prod. Res., 53(6), pp. 1736-1745 (2015).

8. Zaretalab, A., Haghighi, H.S., Mansour, S., et al. "A mathematical model for the joint optimization of machining conditions and tool replacement policy with stochastic tool life in the milling process", Int. J. Adv. Manuf. Technol., 96(5-8), pp. 2319-2339 (2018).

9. Diaz, N., Pascual, R., Ruggeri, F., et al. "Modelling age replacement policy under multiple time scales and stochastic usage profiles", Int. J. Prod. Econ., 188, pp. $22-28$ (2017).

10. Chand, S., McClurg, T., and Ward, J. "A model for parallel machine replacement with capacity expansion", Eur. J. Oper. Res., 121(2000), pp. 519-531 (2000).

11. Wang, K.S., Tsai, Y.T., and Lin, C.H. "A study of replacement policy for components in a mechanical system", Reliab Eng Syst Safe., 58(3), pp. 191-199 (1997).

12. Sheikh, A.K., Raouf, A., Sekerdey, U.A., et al. "Optimal tool replacement and resetting strategies in automated manufacturing systems", Int. J. Prod. Res., 37(4), pp. 917-937 (1999).

13. Hashemi, S.T., Ebadati, O.M., and Kaur, H. "A hybrid conceptual cost estimating model using ANN and GA for power plant projects", Neural Comput \& Applic., 31, pp. 2143-2154 (2019).

14. Seo, K. "A methodology for estimating the product life cycle cost using a hybrid GA and ANN model", Artificial Neural Networks - ICANN 2006 The Series Lecture Notes in Computer Science, 4131, Kollias et al., Eds., pp. 386-395 (2006).

15. Firouzi, A. and Rahai, A. "An integrated ANN-GA for reliability based inspection of concrete bridge decks considering extent of corrosion-induced cracks and life cycle costs", Scientia Iranica, 19(4), pp. 974-981 (2012).
16. Al-Chalabi, H.S., Lundberg, J., Al-Gburi, M., et al. " Model for economic replacement time of mining production rigs including redundant rig costs", $J$. Qual. Maint. Eng., 21(2), pp. 207-226 (2015).

17. Aldhubaib, H.A. and Salama, M.A. "A novel approach to investigate the effect of maintenance on the replacement time for transformers", IEEE T. Power Deliver., 29(4), pp. 1603-1612 (2014).

18. Liu, Y., Li, Y., Huang, H., et al. "Optimal preventive maintenance policy under fuzzy Bayesian reliability assessment environments", IIE Trans., 42(10), pp. 734-745 (2010).

19. Fukuda, T. and Shibata, T. "Theory and applications of neural networks for industrial control system", IEEE T. Ind. Electron., 39(6), pp. 472-489 (1992).

20. Holland, J.H., Adaptation in Natural and Artificial Systems: an Introductory Analysis with Applications to Biology, Control, and Artificial Intelligence, University of Michigan Press, Ann Arbor, Mich, USA (1975).

21. Holland, J.H. "Genetic algorithms", Sci Am., 267, pp. 44-50 (1992).

22. Barlow, R.E. and Hunter, L. "Optimal preventive maintenance policies", Operat Res., 8, pp. 90-100 (1960).

23. Handlarski, J. "Mathematical analysis of preventive maintenance schemes", J. Oper. Res. Soc., 31(3), pp. 227-237 (1980).

24. Ahmad, R. and Kamaruddin, S. "An overview of timebased and condition-based maintenance in industrial application", Comput Ind Eng., 63(1), pp. 135-149 (2012).

25. Zhang, J., Tan, J., Liu, X., et al. "Energy saving curing and precision manufacturing technology of tires", AIP Conference Proceedings., 1713, pp. 130002-130004 (2016).

26. Angelova, M. and Pencheva, T. "Tuning genetic algorithm parameters to improve convergence time", IJChER, Article ID 646917 (2011).

27. Tian, X. and Yuan, S. "Genetic algorithm parameters tuning for resource-constrained project scheduling problem", AIP Conf. Proc., 1955(040059), pp. 1-9 (2018).

28. Leung, Y.W. and Wang, Y. "An orthogonal genetic algorithm with quantization for global numerical optimization", IEEE Trans. Evol. Comput., 5(1), pp. 4153 (2001).

29. Rad, M.A.A. and Rad, M.S.A. "Comparison of artificial neural network and coupled simulated annealing based least square support vector regression models for prediction of compressive strength of high-performance concrete", Sci. Iran., 24(2), pp. 487-496 (2017).

30. Timothy, M., Practical Neural Network Recipes in $C++$, Academic Press, p. 174, Toronto (1993). 
31. Pontes, F.J., Amorim, G.F., Balestrassi, P.P., et al. "Design of experiments and focused grid search for neural network parameter optimization", Neurocomputing, 186, pp. 22-34 (2016).

32. Lahiri, S.K. and Ghanta, K.C. "Artificial neural network model with the parameter tuning assisted by a differential evoluation technique: the study of the holdup of the slurry flow in a pipeline", Chem. Ind. Chem. Eng. Q., 15(2), pp. 103-117 (2009).

33. Jeong, C., Min, J.M., and Kim, M.S. "A tuning method for the architecture of neural network models incorporating GAM and GA as applied to bankruptcy prediction", Expert Syst Appl., 39, pp. 3650-3658 (2012).

34. Monks, J.G., Operations Management: Theory and Problems, 3th Edn., McGraw-Hill, New York (1987).

35. Witt, S.F. and Witt, C.A., Modeling and Forecasting Demand in Tourism, Academic Press, Londra (2000).

36. Lewis, C.D., Industrial and Business Forecasting Methods, Butterworths Publishing, Londra (1982).

\section{Biographies}

Seda Hatice Gökler received the BE degree in Industrial Engineering from Eskişehir Osmangazi University, Eskișehir, Turkey and $\mathrm{ME}$ and $\mathrm{PhD}$ degrees in Industrial Engineering from Sakarya University, Sakarya, Turkey. She is currently a Research Assistant in Industrial Engineering at Kahramanmaraş Sütçü İmam University. Her researches focus on statistical process control, quality management, inventory management, artificial intelligence, and machine learning.

Semra Boran received the BE and $\mathrm{ME}$ degrees in Industrial Engineering from Istanbul Technical University, Istanbul, Turkey and $\mathrm{PhD}$ degree in Industrial Engineering from Yıldız Technical University, Istanbul, Turkey. She is currently Professor at the Department of Industrial Engineering, Sakarya University. Her current research interests include quality management, statistical process control, experimental design, and soft computing methods. 